\title{
COVID-19: Current status, Challenges and Future Perspectives
}

\author{
Rajendra Prasad ${ }^{1}$
}

Published online: 9 September 2020

(C) Association of Clinical Biochemists of India 2020

Coronavirus disease 2019 (COVID-19) was emerged in the Wuhan City, Hubei Province of Central China in December 2019 and subsequently has rapidly spread to most of the countries worldwide [1]. To date (2nd August, 2020), Covid-19 caused 17,680,523 confirmed cases and 680,894 deaths in 216 countries as per WHO report as well as also declared as a Public Health Emergency of International Concern (PHEIC). Strikingly, no vaccine as well as effective therapeutic drugs are available in the International market. In view of these facts, there is an urgent need for a better understanding of SARS-COV-2 virus [severe acute respiratory syndrome; previously known as 2019 novel coronavirus (2019-nCoV)] in order to develop any effective vaccines or molecules against COVID-19.

Coronaviruses (CoVs) are positive sense, single stranded RNA viruses of the family coronaviridae that infect a wide host range to produce disease ranging from common cold to severe/fatal illness [2]. The SARS-COV-2 genome consists of $30 \mathrm{~kb}$ in size encodes a large non-structural polyprotein $(\mathrm{ORF} 1 \mathrm{a} / \mathrm{b})$ which is further proteolytically cleaved to generate $15 / 16$ proteins, 4 structural proteins and 5 accessory proteins. The four structural proteins consists of the spike (S) surface glycoprotein, the membrane (M) protein, the envelope (E) protein and the nucleocapsid (N) protein which are essential for SARS-CoV-2 assembly and infections [3, 4]. The spike surface glycoprotein plays a key role in its attachment to host cells. The receptor binding domain (RBD) of the S1 subunit undergo as hinge

Rajendra Prasad

fateh1977@yahoo.com

1 Department of Biochemistry, M.M. Institute of Medical Sciences and Research (MMIMSR), Maharishi

Markandeshwar University (MMU), Mullana, Ambala, India like conformational transition which led to hide or expose the determinants of receptor binding $[5,6]$. $\mathrm{S}$ protein is considered the major target for designing $\mathrm{CoV}$ antiviral therapies, For instances, $\mathrm{S}$ protein inhibitors, $\mathrm{S}$ cleavage inhibitors, neutralizing antibodies, RBD-ACE-2 blockers, Si RNA fusion core blockers and protease inhibitors [7].

Presently, Scientists are actively working towards the development of suitable vaccine candidate and effective therapeutic molecules for controlling the deadly Covid-19 owing to no effective vaccine or specific antiviral drugs against it. Vaccine Research Center (VRC) of the National Institute of Allergy and Infectious Disease (NIAID), National Institute of Health (NIH) are developing a vaccine candidate expressing SARS-CoV-2 S protein in the mRNA vaccine platform technology which is under clinical trials [8]. Passive immunization through direct administration of monoclonal antibodies (mAbs) may play an effective role in $\mathrm{CoV}$ control as an intervention in exposed individuals. It has been observed that patients recovering from SARS display potent neutralizing antibody response [9].

The main measures in clinical management are alleviating clinical symptoms and supportive cares. These are many anti-CoV agents which are mostly pre-clinical molecules. However, some of them are in phase III trials for Covid-19, including remdesivir, ritonavir, and cobicistat [10]. The RNA-dependent RNA polymerase (RdRp) sequence of SARS-CoV-2 has 96\% homology to that of SARS-CoV. It is very critical observation since drugs are developed for SARS-CoV RdRp might have similar efficacy for SARS-CoV-2 RdRp.

In the present scenario, the development of new therapeutic molecules for COVID-19 is not feasible option owing to long time process. Thereby, second option is to use repurpose broadly acting antiviral drugs because of easy availability known pharmacokinetic and 
pharmacodynamic properties, side effects as well as established dosing regimens. The data obtained from recent study against COVID-19 are promising since the drugs remdesivir and Chloroquine were found to be effective.

Achievements in the development of vaccines and therapeutic agents will facilitate the development of effective vaccines and therapeutics against the rapidly spreading COVID-19 infection. Therefore, in current scenario, we need to rely exclusively on preventive measures since considerable time is needed before efforts to develop a new vaccine or antiviral molecules.

Nothwithstanding, the scientists and clinicians are making all the efforts to ameliorate prevention, treatment and control of COVID-19. Moreover, there is an urgent need to conduct the research into the use of SARS-Cov-2 in suitable animal models for analyzing replication, transmission and pathogenesis. Uphill task remains in identifying the exact source of COVID-19 virus, immunological basis of COVID-19 immune responses and whether the mutation prone positive sense ssRNA virus of COVID-19 will become endemic or alters into more lethal forms in near future.

\section{References}

1. Mitra P, Misra S, Sharma P. COVID-19 Pandemic in India: what lies ahead. Ind J Clin Biochem. 2020;35:257-9. https://doi.org/ 10.1007/s12291-020-00886-6.

2. Lingeswaran M, Goyal T, Ghosh R, et al. Inflammation, immunity and immunogenetics in COVID-19: a narrative review. Ind $\mathrm{J}$ Clin Biochem. 2020;35:260-73. https://doi.org/10.1007/s12291020-00897-3.
3. Chan JF, Kok KH, Zhu Z, et al. Genomic characterization of the 2019 novel human-pathogenic coronavirus isolated from a patient with atypical pneumonia after visiting Wuhan [published correction appears. Emerg Microbes Infect. 2020;9(1):221-36. https://doi.org/10.1080/22221751.2020.1719902.

4. Yuan Y, Cao D, Zhang Y, et al. Cryo-EM structures of MERS$\mathrm{CoV}$ and SARS-CoV spike glycoproteins reveal the dynamic receptor binding domains. Nat Commun. 2017;8:15092. https:// doi.org/10.1038/ncomms15092.

5. Wrapp D, Wang N, Corbett KS, et al. Cryo-EM structure of the 2019-nCoV spike in the prefusion conformation. Science. 2020;367(6483):1260-3. https://doi.org/10.1126/science. abb2507.

6. Gui M, Song W, Zhou H, et al. Cryo-electron microscopy structures of the SARS-CoV spike glycoprotein reveal a prerequisite conformational state for receptor binding. Cell Res. 2017;27(1):119-29. https://doi.org/10.1038/cr.2016.152.

7. Du L, He Y, Zhou Y, Liu S, Zheng BJ, Jiang S. The spike protein of SARS-CoV-a target for vaccine and therapeutic development. Nat Rev Microbiol. 2009;7(3):226-36. https://doi.org/10. 1038/nrmicro2090.

8. Moderna. Moderna Announces Funding Award from CEPI to Accelerate Development of Messenger RNA (mRNA) Vaccine Against Novel Coronavirus 2020 (accepted 2020 Feb 15). https:// investors.modernatx.com/news-releases/news-release-details/ moderna-announces-funding-award-cepi-acceleratedevelopment.

9. Yang ZY, Kong WP, Huang Y, et al. A DNA vaccine induces SARS coronavirus neutralization and protective immunity in mice. Nature. 2004;428(6982):561-4. https://doi.org/10.1038/ nature 02463 .

10. Morse JS, Lalonde T, Xu S, Liu WR. Learning from the past: possible urgent prevention and treatment options for severe acute respiratory infections caused by $2019-\mathrm{nCoV}$. ChemBioChem. 2020;21(5):730-8. https://doi.org/10.1002/cbic.202000047.

Publisher's Note Springer Nature remains neutral with regard to jurisdictional claims in published maps and institutional affiliations. 